\title{
Access Point Backhaul Resource Aggregation as a Many-to-One Matching Game in Wireless Local Area Networks
}

\author{
Kawther Hassine, ${ }^{1}$ Mounir Frikha, ${ }^{1}$ and Tijani Chahed ${ }^{2}$ \\ ${ }^{1}$ Higher School of Communications of Tunis (Sup'Com), Tunis, Tunisia \\ ${ }^{2}$ Institut Mines-Telecom, Telecom SudParis, Paris, France \\ Correspondence should be addressed to Kawther Hassine; kaouther.hassine@supcom.tn
}

Received 22 September 2016; Revised 9 March 2017; Accepted 10 April 2017; Published 15 May 2017

Academic Editor: Michael McGuire

Copyright (c) 2017 Kawther Hassine et al. This is an open access article distributed under the Creative Commons Attribution License, which permits unrestricted use, distribution, and reproduction in any medium, provided the original work is properly cited.

\begin{abstract}
This paper studies backhaul bandwidth aggregation in the context of a wireless local area network composed of two different types of access points: those with spare backhaul capacity (which we term providers) and those in shortage of it (beneficiaries); the aim is to transfer excess capacity from providers to beneficiaries. We model the system as a matching game with many-to-one setting wherein several providers can be matched to one beneficiary and adopt the so-called deferred acceptance algorithm to reach an optimal and stable solution. We consider two flavors, when the beneficiaries are limited in their resource demands and when they are not, and two scenarios, when resources are abundant and when they are scarce. Our results show that the many-to-one setting outperforms the one-to-one case in terms of overall throughput gain, resource usage, and individual beneficiaries satisfaction by up to $50 \%$, whether resources are scarce or abundant. As of the limited versus nonlimited case, the former ensures more fair sharing of spectral resources and higher satisfaction percentage between beneficiaries.
\end{abstract}

\section{Introduction}

The current and recent trends in mobile networks are towards exponential increase in the use of spectral resources. Although current capacity requirements seem to be containable, bandwidth consumption will continue to increase with future generations of wireless networks.

In the context of a heterogeneous small cell-based architecture, there are three basic areas of connectivity, namely, Personal Area Networks (PANS), wireless local area networks (WLANs), and cellular coverage. Under this umbrella, small cell-based mobile networks are principally used to bridge connections to the device by means of capillary networks. Meanwhile, traffic offload and local access is often covered by means of a short-range wireless technology.

In this context, rather than deploying their own edging sites, operators trends are towards more efficient and costeffective solutions. The latter include using WiFi in the last mile coverage. In fact, WiFi WLANs will be responsible for offloading traffic from and to macrocells by traffic amounts up to $52 \%$ by 2018 (source: Cisco VNI Global Mobile Data
Traffic Forecast 2013-2018). Besides, voice traffic lost from LTE systems to WiFi networks will reach $53 \%$ by 2020 (source: Cisco VNI Mobile, 2016).

From this perspective, pressure is increasing on existing WiFi backhaul segments. This portion of the network that starts at the radio access network and ends in the mobile core network (see Figure 1) is the key answer to sustain traffic growth and relieve pressure off both mobile and wireless networks. This is where new ways to manage backhaul resources need to be considered.

In the context of wireless local area networks, a major research trend targets better management of the available backhaul resource at access points (APs) through the socalled backhaul bandwidth aggregation. With no major short-term investments and no altering to network topology, this technique enables wireless clients to connect simultaneously to different APs and cumulate available backhaul capacities in order to improve their transmission rates (see Figure 2).

Backhaul bandwidth aggregation techniques can be broadly classified in two categories: client-oriented and 


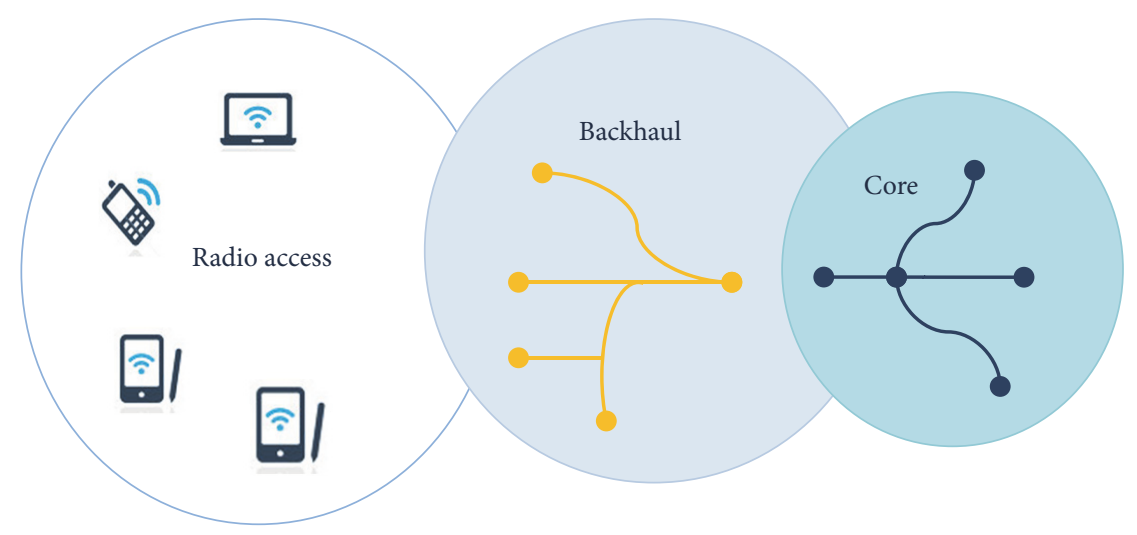

FIGURE 1: Wireless network architecture.

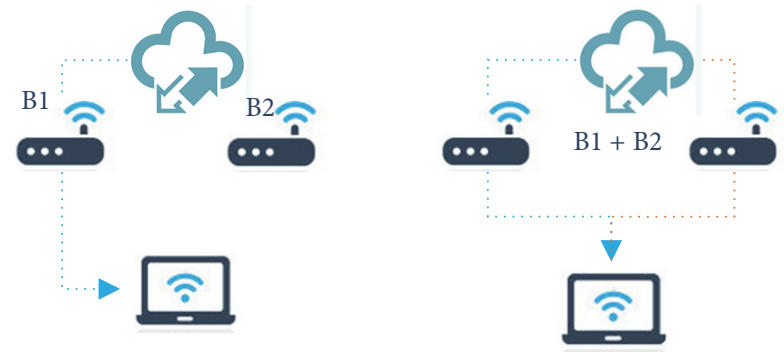

FIgURE 2: AP backhaul aggregation.

AP-oriented. In the former, studies empowered wireless clients as the aggregation initiator, with several sophisticated aggregation mechanisms. Whether protocol, driver, or virtualization mechanisms, these upgrades enabled wireless clients to ask for and, if possible, acquire available shares of backhaul resources from nearby APs [1-3].

As for the AP-oriented approaches, the aggregation process is monopolized by access points. With proper signaling schemes between involved wireless clients, each AP builds its own image of the entire network and then decides how to manage its spare capacity among the entire set of reachable clients $[4,5]$.

Even though these solutions improve network performance in terms of overall throughput, they are still subject to several flaws. On one hand, recursive interactions between wireless clients and access points raise interoperability issues and lead to higher interference level and signaling overload, along with a cost challenging implementation due to the unpredictable nature of involved equipment (stations, smart phones, tablets, etc.).

On the methodological level, most approaches opted for conventional unilateral optimization methods [6] when it comes to backhaul resources allocation. These methods require an overall network description, a centralized control scheme, and a prediction model for the signal propagation. This yields higher complexity levels especially for combinatorial and integer programming problems such as resource allocation and user association [7].

For the aforementioned limitations, we proposed in [8] an alternate solution for backhaul capacity aggregation. Our approach defines a symmetric AP-exclusive protocol (no wireless clients involved) for available backhaul resource (ABR) allocation. The idea consists of dividing first the ensemble of APs into symmetric separate sets: (1) providers (P-AP), with spare backhaul resources, and (2) beneficiaries (B-AP), in need of more backhaul bandwidth. Based on the two-sided nature of our system, we proposed to assign the available backhaul resources using a matching game.

We, however, restricted ourselves in [8] to one-to-one matching wherein each player could be matched with one player at most. In this case, even though B-APs improve their transmission rates, access to backhaul resources was limited in a way that holds back most of them from fulfilling their needs in terms of required backhaul capacity.

In this paper, we propose to extend our work to the case of many-to-one matching, wherein each beneficiary (B-AP) has the right to access as many resources as possible. Providers (P-APs) are, however, still limited to one user at once so as to avoid higher complexity levels and signaling overhead.

The remainder of this paper is organized as follows. In Section 2, we describe our system and model. In Section 3, we formulate our model based on a many-to-one matching game and derive its solution. In Section 4, we display simulation results of the many-to-one matching game for different scenarios. In Section 5, we conclude the paper and give some hints on future research steps.

\section{System and Model}

We consider an Extended Service Set (ESS) in an indoor area with a finite number of IEEE 802.11n capable APs distributed within a " $20 \times 20$ " square area.

Let $\delta$ be the set of infrastructure Basic Services Sets (BSS), each connected to the core network via backhaul links (wired or wireless). Frequency bands in $\mathcal{S}$ correspond to sub-6 GHz Line of Sight (LOS) point-to-point links and are subject to additive white Gaussian noise. In this band, as the antenna beams are much wider [9], the propagation is interference limited [10]. The Signal to Interference plus Noise Ratio (SINR) level is function of the AP's location, transmit power, propagation model of the carrier, and the link state information. 
APs, at the head of each BSS, must share overlapping coverage area. With no spatial diversity involved, each AP handles a random set of primary wireless customers. The status of each AP is determined by the amount of backhaul capacity: either in excess, denoted by $\omega_{n}$, or in shortage of it, denoted by $R_{k}$, once it has served its primary clients. Let $\mathcal{N} \subset \mathcal{S}$ denoting the set of $N$ providing APs be the number of P-APs and $\mathscr{K} \subset \mathcal{S}$ the set of $K$ beneficiary APs.

According to our proposal, P-APs are willing to cede their available backhaul capacity to the rest of B-APs on a competitive basis between the latter. Here, $\mathcal{N}$ represents an $A B R$ equal to the ensemble of all spare backhaul bandwidth at each P-AP:

$$
\mathrm{ABR}=\left\{\omega_{1}, \omega_{2}, \ldots, \omega_{n}, \ldots, \omega_{N}\right\} .
$$

For the described resource allocation problem, our purpose is to find optimal ABR allocation over P-APs $\subset \mathcal{N}$ to serve B-APs $\subset \mathscr{K}$ so as to maximize the overall throughput of beneficiary APs (see Figure 3).

The individual throughput gain at one given $\mathrm{B}-\mathrm{AP}_{k}$ is in fact the achievable rate $r_{n k}$, determined by the following equation $[11,12]$ :

$$
r_{n k}=\omega_{n} \log _{2}\left(1+\gamma_{n k}\right),
$$

where

$$
\gamma_{n k}=\frac{\psi_{n}\left|h_{k n}\right|^{2}}{\sum_{j \in \mathscr{K} \backslash k} \psi_{j}\left|h_{j k}\right|^{2}+\sigma^{2}} .
$$

Here, $\gamma_{n k}$ is the SINR between B-AP ${ }_{k}$ and ${\mathrm{P}-\mathrm{AP}_{n}}_{n}$ [13]. $h_{k n}$ (resp., $h_{n k}$ ) is the Channel State Information at the Transmitter (CSIT) (resp., at the receiver, CSIR), estimated and quantized at the radio link between $\mathrm{B}^{-\mathrm{AP}_{k}} \in \mathscr{K}$ and $\mathrm{P}_{-\mathrm{AP}_{n}} \in \mathcal{N}$.

$\psi_{n}$ and $\sigma^{2}$ are the transmit power of a given $\mathrm{P}_{-} \mathrm{AP}_{n} \in \mathcal{N}$ and the variance of the receiver's Gaussian noise, respectively.

Considering a Time Division Multiple Access (TDMA) to resources, we propose to assign one P-AP's backhaul resource to only one B-AP at once. On the other side, B-APs could have access to all backhaul resources within reach. This defines a many-to-one association between P-APs and B-APs with the aim of maximizing the following function:

$$
\begin{array}{ll}
\max & R_{\text {total }}\left(r_{n k}, \eta_{n k}\right) \\
\text { s.t. } & r_{n k}\left(\eta_{n k}, \gamma_{n k}\right) \leq \omega_{n}, \quad \forall n \in \mathscr{N} \\
& \sum_{n \in \mathcal{N}} \eta_{n k} \leq N, \quad \forall k \in \mathscr{K} \\
& \sum_{k \in \mathscr{K}} \eta_{n k} \leq 1, \quad \forall n \in \mathscr{N} \\
& \eta_{n k} \in\{0,1\} .
\end{array}
$$

Here, $R_{\text {total }}\left(r_{n k}, \eta_{n k}\right)$ is the overall throughput gain at the $\mathrm{B}-\mathrm{APs}$, expressed as follows:

$$
R_{\text {total }}\left(r_{n k}, \eta_{n k}\right)=\sum_{k \in \mathscr{K}} \sum_{n \in \mathscr{N}} \eta_{k n} r_{n k}
$$

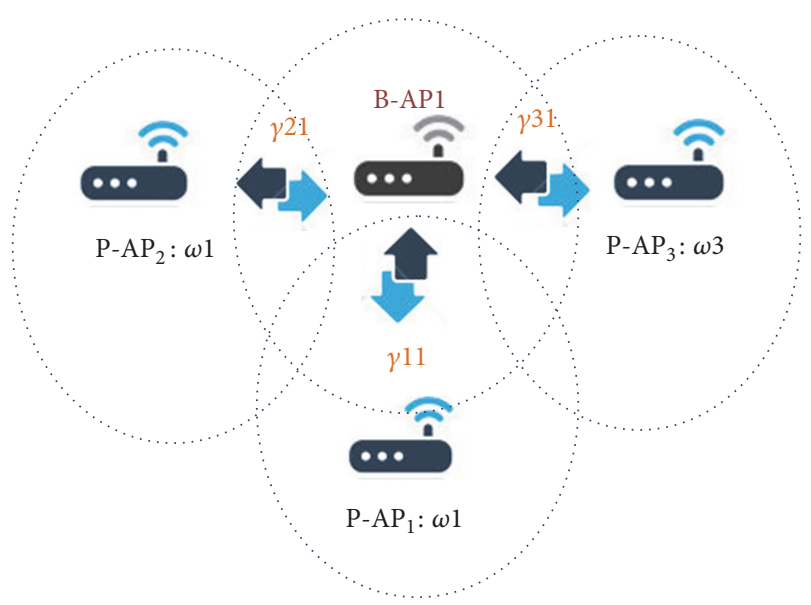

Figure 3: AP-to-AP aggregation scheme.

where $r_{n k}$ is the achievable rate at $\mathrm{B}-\mathrm{AP}_{k}$ from $\mathrm{P}-\mathrm{AP}_{n} \cdot \omega_{n}$ is the corresponding $\mathrm{ABR}$ and $\eta_{n k}$ is the $\mathrm{ABR}$ allocation variable, such that $\eta_{n k}=1$ if $\mathrm{P}_{-\mathrm{AP}_{n}} \in \mathcal{N}$ allocates its $\mathrm{ABR}$ to $\mathrm{B}_{-} \mathrm{AP}_{k}$ and $\eta_{n k}=0$, otherwise.

And, so, the achievable backhaul rate for $\mathrm{B}_{-} \mathrm{AP}_{k}$ can be written as follows:

$$
r_{n k}= \begin{cases}\omega_{n} \log _{2}\left(1+\gamma_{n k}\right) & \text { if } \eta_{n k}=1 \\ 0 & \text { if } \eta_{n k}=0,\end{cases}
$$

where $\gamma_{n k}$ represents the SINR obtained from (3) and $\omega_{n}$ is again the available backhaul resource of $\mathrm{P}-\mathrm{AP}_{n}$.

Taking into account the SINR, which is function of the AP's location, transmit power, propagation model of the carrier, and backhaul resource allocation, we can translate the optimization problem in (4) and (5) into an assignment problem where the available backhaul resource of a P-AP $\in \mathcal{N}$ is assigned to one given $\mathrm{B}-\mathrm{AP} \in \mathscr{K}$. This problem is a $0-1$ integer programming which is a satisfiability problem such as the one of Karp's $21 \mathrm{NP}$-complete problems, known to be difficult to solve via classical optimization approaches [14].

With the two-sided nature of our system [15] (P-AP versus $\mathrm{B}-\mathrm{AP})$, we propose to address this problem with a distributed approach, as shown next.

\section{AP Backhaul Aggregation as a Many-to-One Matching Game}

3.1. Problem Formulation. A matching game is a two-side assignment problem. Each side represents a separate set of players seeking for their best match among the players of the other set.

In this framework, each beneficiary AP identifies its potential match among the providing APs and then initiates a selection process, as shown in Figure 4. This selection process is based on a preference relationship defined for each side, which we explicit next.

Definition 1. A preference relationship $>$ is defined as a complete, reflexive, and transitive binary relationship between elements of a given set. 


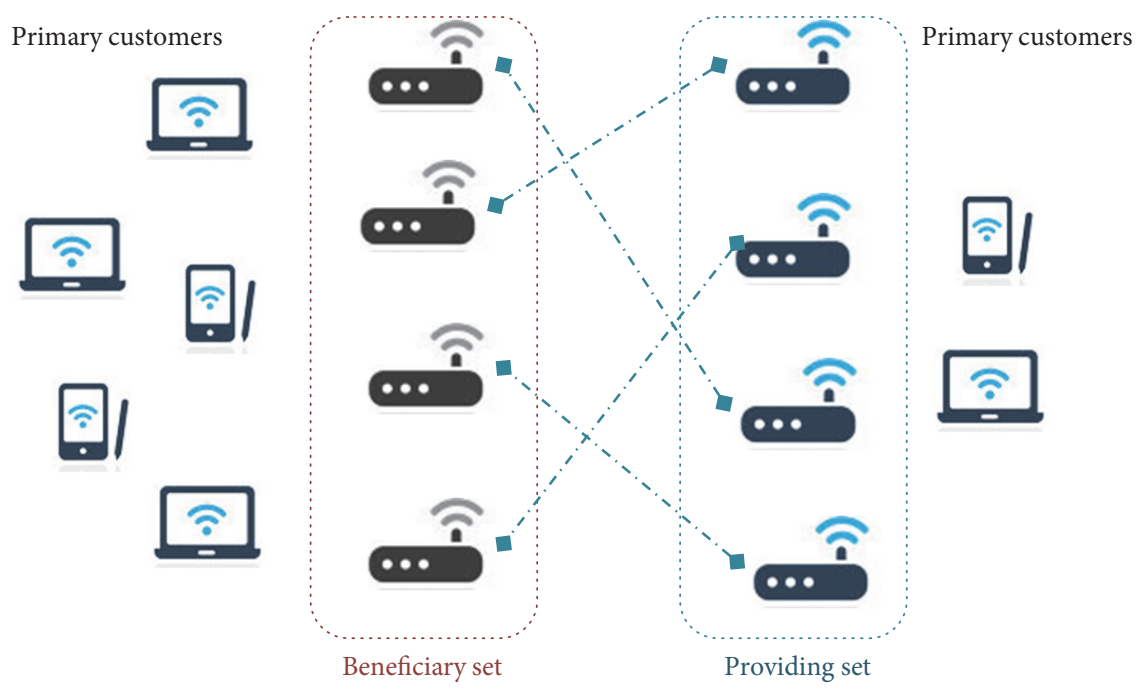

FIGURE 4: ABR allocation as a matching problem.

In our context, we consider $>_{k}$ as the preference relationship of a $\mathrm{B}-\mathrm{AP}_{k} \in \mathscr{K}$ over the set of backhaul resources of $\mathrm{P}$-APs $\subset \mathcal{N}$. We denote $\mathrm{P}-\mathrm{AP}_{n 1}>_{k} \mathrm{P}_{-} \mathrm{AP}_{n 2}$ if $\mathrm{B}^{-\mathrm{AP}_{k}}$ prefers $\mathrm{P}-\mathrm{AP}_{n 1}$ to $\mathrm{P}-\mathrm{AP}_{n 2}$ given their respective available backhaul resources.

The preference of players (B-APs) over a set of choices (P-APs) is a decisive factor when it comes to the ultimate matching map. We refer here to a particular ordering of $A B R$ resources based on their relative outcome or utility.

We define individual preference relationships using utility functions for B-APs and P-APs. And usually there is not a single utility function associated with one $\mathrm{ABR}$, but rather an overall utility function $U$ defined to describe the outcome of the whole network, for example, the sum utility or proportional fair utility. Assuming our players (here we refer to APs) to be rational, as they are choosing to maximize their own payoffs, we define $a>_{k} b \Leftrightarrow U(a)>U(b)$.

For the studied backhaul management problem, the preference of each player is crucial when it comes to the selection process [16] especially if it is restricted to a quota: we refer here to the number of simultaneous physical matches a player is allowed.

In a one-to-one matching, players quotas prevented BAPs from achieving their required capacities. Even if extra resources are involved, B-APs wasted valuable opportunities to increase their outcome. To remedy this situation we propose to rise B-APs quota, $q_{k}$, in terms of accessible ABR to $N$.

And so, we introduce a two-sided many-to-one canonical matching game where P-APs are committed to cede their available backhaul capacity to the rest of B-APs. A many-toone matching can formally be described as follows [17].

Definition 2. Given the two disjoint finite sets of players $\mathscr{K}$ and $\mathcal{N}$, a matching game $\mu$ is defined as a function from $\mathscr{K}: \rightarrow \mathcal{N}$ from which we have the following:

(1) $\forall n \in \mathscr{N}, \mu(n) \in \mathscr{K}$.
(2) $\forall k \in \mathscr{K}, \mu(k) \subseteq \mathcal{N}$.

(3) $\mu(n)=k$ if and only if $n \in \mu(k)$.

Accordingly, we have $\mathrm{P}_{-} \mathrm{AP}_{n} \in \mu(k)$, if $\mathrm{P}_{-} \mathrm{AP}_{n}$ is assigned to $\mathrm{B}-\mathrm{AP}_{k}$ through a matching $\mu$ and $\mathrm{P}-\mathrm{AP}_{n} \notin \mu(k)$, otherwise. The purpose of each B-AP $\in \mathscr{K}$ is to find its perfect match(es) $\mu^{*}$, enabling it to achieve a maximal outcome, while respecting the P-APs available capacity. The ultimate matching aims to maximize the overall throughput. And so, we have again the following:

$$
\begin{aligned}
\max & R_{\text {total }}\left(r_{n k}, \eta_{n k}\right) \\
\text { s.t. } & r_{n k}\left(\eta_{n k}, \gamma_{n k}\right) \leq \omega_{n}, \quad \forall n \in \mathcal{N} .
\end{aligned}
$$

Equations (9) imply that the outcome of a B-AP is measured in terms of achievable throughput. In this case, the preference relationship of B-APs depends on link state information along with available backhaul bandwidth $\{\omega\}_{j=1 \cdots N}$, at P-AP's level. Adequate utility functions, reflecting players preference on each side, are to be defined.

Let $V_{k, n(\cdot)}$ and $U_{n, k}(\cdot)$ denote, respectively, the utility functions of players in $\mathscr{K}$ and $\mathcal{N}$. For a given $\mathrm{B}_{-} \mathrm{AP}_{k}$, we define $S_{k}$ to be the set of available P-APs (within reach). Each BAP will be looking for the P-APs that provide the best overall achievable rate. So, a $\mathrm{B}-\mathrm{AP}_{k} \in \mathscr{K}$ will yield a utility $V_{k, n}$ to

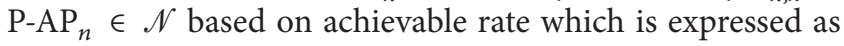
follows:

$$
V_{k, n}\left(\gamma_{k n}\right)=\omega_{n} \log _{2}\left(1+\gamma_{k n}\right) .
$$

From the opposite side, an access point from the providing set aims to allocate its $\mathrm{ABR}$ to the most profitable $\mathrm{B}-\mathrm{AP}_{k} \in \mathscr{K}$ (whose achievable rate will be maximized).

Actually, both P-APs and B-APs agree on the same profit. And so, the utility function of a $\mathrm{P}-\mathrm{AP}_{n} \in \mathscr{N}$ over B-APs is given by the following:

$$
U_{n, k}\left(\gamma_{n k}\right)=\omega_{n} \log _{2}\left(1+\gamma_{n k}\right) .
$$


Input. $\mathscr{K}, \mathcal{N}, \mathrm{ABR}=\left\{\omega_{n}\right\}_{n \in \mathcal{N}}$

Initialize $S_{k}$ to be the total available P-APs for a given B-AP Initialize $q_{k}$

Stage 1

(1) B-APs rank all P-APs using $V_{k, n}$

(2) Every B-AP $k_{k}$ applies for its most preferred P-AP $n^{*}$ from $S_{k}$ and removes $\mathrm{P}_{-} \mathrm{AP}_{n^{*}}$ from $S_{k}$

Stage 2

(1) P-APs rank each applicant using $U_{n, k}$

(2) Among all applicants and its current match $\mu(n)$, $\mathrm{P}-\mathrm{AP}_{n}$ accepts the most preferred $\mathrm{B}-\mathrm{AP}_{k^{*}}$ and rejects the others.

If $\mathrm{B}-\mathrm{AP}_{k}$ is accepted by $\mathrm{P}-\mathrm{AP}_{n}$ then:

$r_{n k}=r_{n k}+\omega_{n} \log _{2}\left(1+\gamma_{n k}\right),|\mu(k)|=|\mu(k)|+1$

If ${\mathrm{B}-\mathrm{AP}_{k}}_{k}$ is rejected by $\mathrm{P}_{-\mathrm{AP}_{n}}$, then:

$$
r_{n k}=r_{n k},|\mu(k)|=|\mu(k)|
$$

If $\mathrm{B}-\mathrm{AP}_{k}$ is accepted then rejected by $\mathrm{P}-\mathrm{AP}_{n}$, then:

$r_{n k}=r_{n k}-\omega_{n} \log _{2}\left(1+\gamma_{n k}\right),|\mu(k)|=|\mu(k)|-1$

Stage 3. Repeat Stage 1 and Stage 2 for every $\mathrm{B}-\mathrm{AP}_{k} \in \mathscr{K}$

until

$$
S_{k}=\emptyset \text { or }|\mu(k)| \geq q_{k}
$$

Stage 4. Exchanged traffic will be forwarded from $\mathrm{B}-\mathrm{AP}_{k}$ through its respective match $\mathrm{P}-\mathrm{AP}_{n} \in \mu\left(\mathrm{B}-\mathrm{AP}_{k}\right)$

Output. Stable optimal matching $\mu^{*}$

Algorithm 1: DA algorithm user proposing: many-to-one matching.

Once our model of a many-to-one matching game is identified, our next step would be to introduce a solution that ensures a stable and optimal matching among different sets of APs.

3.2. Deferred Acceptance Algorithm as a Solution for the Matching Game. In a matching game, the major question is whether we can find a stable matching for all players involved in the game. Such issue was treated and positively answered in [17]. In fact, each matching problem has at least one stable matching thanks to the so-called deferred acceptance (DA) algorithm; this matching is also shown to be optimal.

There are two versions for the DA algorithm: user proposing versus resource proposing. Usually, the proposing set is more highly regarded since the outcome is more likely to be preferable to that set. We give precedence in this work to B-APs, because P-APs (and their wireless primary clients) are already satisfied in terms of resources, with or without matching.

The DA algorithm user proposing of a many-to-one matching is detailed in Algorithm 1.

Prior to the DA algorithm, the B-APs obtain the channel state from the control signals sent by the P-APs, along with their available backhaul capacity $\omega_{n}$.

As a start, each B-AP broadcasts its status as beneficiary $\mathrm{AP}$ and then scans the wireless medium for potential P-APs. Accordingly, each B-AP $\mathrm{A}_{k}$ initializes its quota $q_{k}=N$ and the set $S_{k}=\mathscr{N}$ as the list of all P-APs B-AP $k$ may apply to.

In Stage 1, the B-APs rank the P-APs based on their

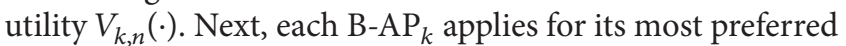
$\mathrm{P}-\mathrm{AP}_{n^{*}}=\operatorname{argmax} V_{k, n}(k, n)$ from the set $S_{k}$, as long as its current matching size $|\mu(k)|$ does not exceed its quota. And then, it removes $\mathrm{P}-\mathrm{AP}_{n^{*}}$ from its preference list, in order to avoid applying for the same P-AP multiple times.

In Stage 2, the P-APs receive the applications and calculate the corresponding utility values using $U_{n, k}(\cdot)$. Each P-AP accepts the most preferred $\mathrm{B}-\mathrm{AP}_{k^{*}}=\operatorname{argmax} U_{n, k}(n, k)$ and rejects previous $\mu(n)$ as well as the rest of the applicants. If accepted by $\mathrm{P}_{-} \mathrm{AP}_{n}, \mathrm{~B}-\mathrm{AP}_{k}$ gets to add the achievable rate from that link to its current rate. In case where $\mathrm{B}-\mathrm{AP}_{k}$ has been accepted and then rejected by $\mathrm{P}-\mathrm{AP}_{n}, \mathrm{~B}-\mathrm{AP}_{k}$ subtracts the corresponding achievable rate from its total gain.

The algorithm terminates within a finite number of iterations: either when all users reach their quotas or when the list of preferences is drained: $S_{k}=\emptyset$ (every unmatched user has been rejected by every possible resource). The final stage reflects a stable matching where we can ensure that all B-APs are mapped to their optimal match(es).

Once matched, $\mathrm{B}-\mathrm{AP}_{k}$ no longer forwards its excess traffic over its own backhaul link. In fact, $\mathrm{P}$-APs $\in \mu^{*}(k)$ grant $\mathrm{B}-\mathrm{AP}_{k}$ a public access to their backhaul medium over the radio channel. The excess traffic will be forwarded from $\mathrm{B}-\mathrm{AP}_{k}$ to $\mathrm{P}-\mathrm{AP}_{n} \in \mathcal{N}$ over the $\mathrm{ABR} \in \mu^{*}(k)$. Access to these resources is scheduled in TDMA basis, according to the preference order.

Accordingly, the B-AP gets the sum of the acquired backhaul capacities as a potential throughput gain. This case is applicable if the wireless capacity of the B-AP exceeds the offered backhaul capacity. Otherwise, the effective transmission rate will be ceiled to the radio channel width.

\section{Results and Discussions}

4.1. Simulation Settings. For our simulations, we consider an ESS in an indoor WLAN area with a finite number of APs. In such configuration, B-APs and P-APs are distributed randomly within a " $20 \times 20$ " square area. The central frequency corresponds to that of the IEEE 802.11n standard, that is, $5 \mathrm{GHz}$. Simulation parameters are summarized in Table 1 .

We simulate network response of the many-to-one matching game in comparison with previously addressed one-to-one matching game [18]. To do so, we consider several " $a \times b$ " configurations where $a$ and $b$ denote, respectively, the number of B-APs and P-APs in each matching set.

Results include illustrative example of optimal matching maps and the overall acquired capacity $((12))$ of $\mathrm{B}-\mathrm{AP}_{k}$ from the set of its matches $\left\{\mathrm{P}-\mathrm{AP}_{j}\right\}_{j \in \mu^{*}(k)}$ :

$$
r_{k}=\sum_{j \in \mu^{*}(k)} r_{j k} .
$$

We show also the ABR consumption in percentage ((13)) and the B-APs' satisfaction percentage (B-APs which reached their threshold limit, $r_{k} \geq R_{k}$ ):

$$
\% \text { of } \mathrm{ABR}=\frac{\sum_{k \in \mathscr{K}} r_{k}}{\sum_{n \in \mathscr{N}} \omega_{n}} .
$$

Depending on the size of opposite sets, we define two possible cases: (1) the "scarce" resource case, if B-APs outnumber 

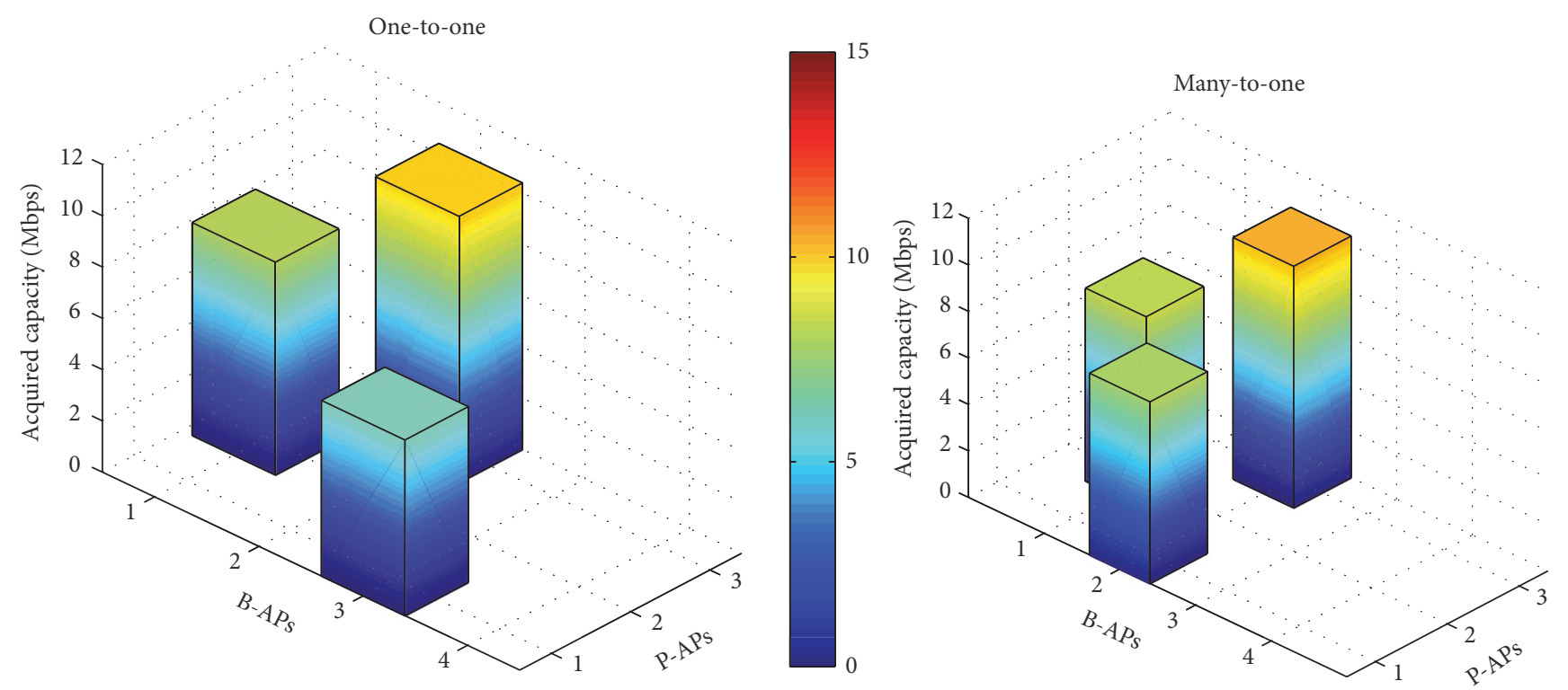

FIGURE 5: One-to-one versus many-to-one optimal mapping: “ $4 \times 3$ ”.

TABLE 1: Simulation parameters.

\begin{tabular}{lcc}
\hline Notation & Parameter & Value \\
\hline $\mathcal{N}$ & Number of P-APs & 3 to 5 \\
$\mathscr{K}$ & Number of B-APs & 3 to 5 \\
$\omega_{n}$ & ABR per P-AP & 5 to $20 \mathrm{Mbps}$ \\
$\sigma^{2}$ & Noise power & 10 to $30 \mathrm{dBm}$ \\
$\psi$ & Transmit power & 80 to $100 \mathrm{~mW}$ \\
\hline
\end{tabular}

P-APs, and (2) the "abundant" resource case, otherwise. Each case will be detailed separately as we proceed.

4.2. Scarce Resource Case. In a scarce resource case $(a>b)$, the matching proposals will not be entirely satisfied: one or more B-APs (the least preferable) will remain unmatched.

Figure 5 shows one particular realization of a matching between P-APs and B-APs for both one-to-one and many-toone allocation modes: “ $4 \times 3$ " configuration.

Figures 6 and 7 show the resulting individual performance in terms of achievable rate $\left\{r_{k}\right\}_{k=1 \cdots 4}$, obtained after acquiring extra resources from the set of optimal matches (given by (8)) in comparison with related thresholds $\left\{R_{k}\right\}_{k=1 \cdots 4}$.

We observe that the mapping between matching sets differs with the allocation scheme. In fact, in a one-to-one matching game with " $a \times b$ " configuration case $(a>b)$, at least $b$ beneficiaries get to be matched, since the quota of each player is ceiled to one (including P-APs). In this case, BAPs have almost equal chances to access available resources. And so, a larger number of B-APs involved in the matching process get a spare backhaul capacity added to their account. Still, in most cases, this one share of ABR is less than enough compared to the required capacity.

Added to that, some matched B-APs could be more of a "destructive" match with degraded link conditions and

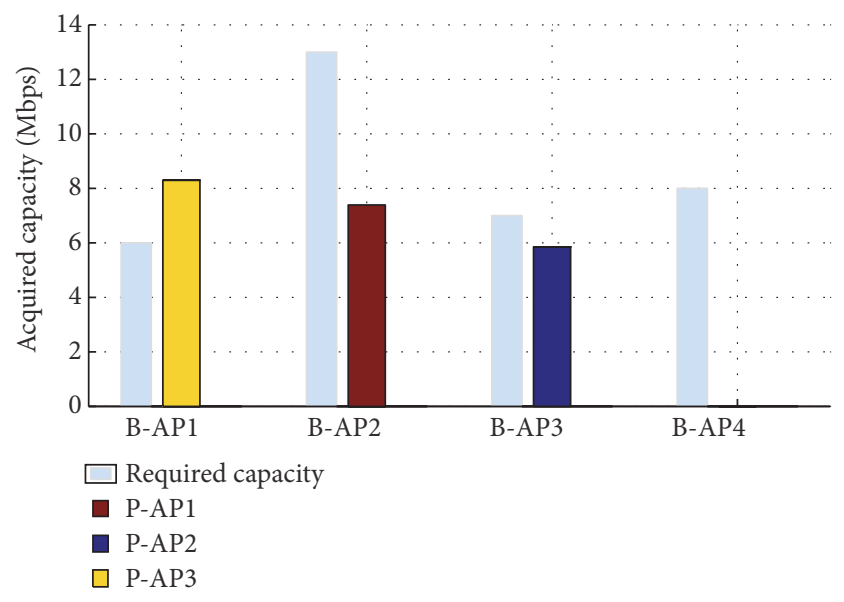

FIGURE 6: One-to-one matching: acquired capacity per B-AP, “ $4 \times 3$ ”.

low SINR (e.g., B-AP3 in Figure 6). And so, serving these B-APs not only curbs network response when it comes to communication quality but also fails to achieve B-APs needs in terms of required capacity (B-AP2: $56 \%$ and B-AP3: 79\%).

In the context of a many-to-one matching, the quota of each beneficiary equals to $N$ : the amount of available resources. Here, none of the P-APs is forced to jump over their first and obviously best choice to reach the remaining of its preference list. And so, each P-AP engages only with privileged B-APs (better SINR) in the queue. This way, PAPs will be able to sustain better signal strength and optimal transmission rates.

On the other hand, free access to available resources enabled more B-APs to reach their required capacity. In this case, better satisfaction levels are ensured (50\% versus $25 \%$ for the one-to-one matching).

Still, one B-AP could theoretically seize a majority of available resources. Most likely, such B-AP would amply 


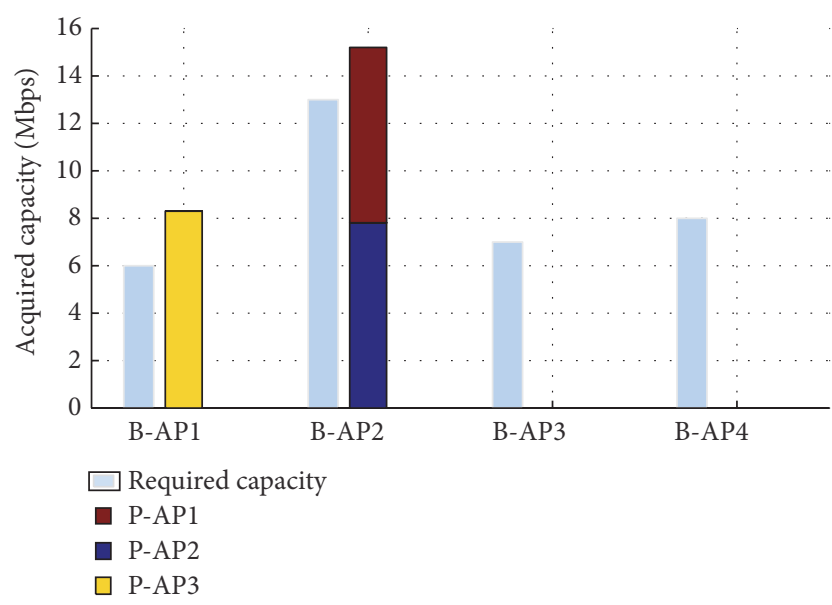

FIgURE 7: Many-to-one matching: acquired capacity per B-AP, “ $4 \times$ 3".

exceed its needs in terms of transmission capacity (e.g., 147\% for B-AP2). Meanwhile, other B-APs are left with no resources at all (B-AP3 in Figure 7).

To remedy this issue, we will present in Section 4.4 one way to curb such B-APs greediness. To do so, access to available resources will be limited by a predetermined threshold beyond which a B-AP withdraws from the selection process.

4.3. Abundant Resource Case. The abundant resource case arises when the number of P-APs exceeds the number of $\mathrm{B}-\mathrm{APs}$; that is, $a \leq b$. Here, we consider a " $3 \times 5$ " configuration as an illustrative example of the abundant resource case.

Figure 8 shows again the many-to-one matching as opposed to the one-to-one matching. The optimal matching shows that, even with extra resources involved, the one-toone rule precludes B-APs from acquiring further throughput, whereas the many-to-one allocation leaves no resource unused.

Detailed results, shown in Figures 9 and 10, prove that many-to-one distribution is more adequate for the abundant resource case.

In the case of one-to-one matching scheme, a quotalimited access to resources holds B-APs from cumulating available backhaul capacity even with extra resources involved. And so, a one-to-one association misses valuable resources on the way and substantially restrains one given BAP from fulfilling its required capacity. In the many-to-one scheme, B-APs with higher SINR levels are free to establish a multiple match including several resources at once. Once satisfied, a B-AP could withdraw from the beneficiary set to make room for other candidates.

We show in Figure 11 the average ABR consumption along with satisfaction numbers of these matching schemes, resulting from 10 realizations of optimal matchings. This illustrates the spectrum efficiency for both approaches.

On one hand, we observe a difference between the oneto-one and many-to-one schemes (from 10 to $40 \%$, resp., for scarce and abundant resource case) in terms of resource consumption. The disparity is due to the partition strategy.
Actually, based on experiments which we do not display here, the gap between the two approaches is larger as the matching sets size gets larger. So, compared to the one-toone matching, the many-to-one matching capitalizes more efficiently on available resources, especially for the abundant resource case.

On the other hand, results show that up to $20 \%$ for the scare resources case and $32 \%$ for the abundant resource case of involved B-APs reach satisfaction in one-to-one scheme, when it comes to required capacity threshold, while up to, respectively, $35 \%$ and $73 \%$ of B-APs in many-to-one context reach or even exceed their threshold of capacity. This way, fulfilled B-APs could be removed from the applicant list, and new matching opportunities arise for the rest of the B-APs. Such feature is crucial for an efficient resource allocation, especially, in the presence of a dynamic variation of APs' status in a WLAN.

To summarize, compared to a one-to-one scheme, the many-to-one scheme fits better network requirements in terms of overall throughput gain (almost 180\% better) and spectral efficiency.

In the one-to-one matching, B-APs have no choice but to get this one "shot" and retire from the game even with abundant resource case. This way, B-APs chances of reaching their individual requirements drop considerably. On the other side, P-APs are in most cases pushed to serve the least preferable B-APs (B-AP3 in Figure 9) knowing that better ones are already taken. This leads to a degraded link quality and lower achievable rates.

In the many-to-one case, access to resources is driven by B-AP's needs and its position on the preference list. These factors push towards two major outputs: (1) higher transmission rate and better link quality, given that better placed B-APs are the one to be served, in priority, and (2) better satisfaction levels (66\%), considering that each B-AP is greedily and relentlessly seeking further backhaul resources as long as they are available.

However, as stated in the previous section as well, such a greedy allocation scheme leads sometimes to unfair allocation of backhaul resources by excluding less fortunate BAP from the selection process. For this, we introduce and evaluate next a limited approach for the many-to-one based ABR allocation.

4.4. Limited versus Greedy ABR Allocation. In a fair, budgetlimited environment, backhaul resources are subject to physical and regulatory restrictions. And so, in order to ensure better fairness, we propose to set a limit on B-APs access to available resources.

In this context, for each B-AP involved in the selection of $\mathrm{ABR}$, we define a limit on its acquired capacity, $r_{k}$, which is equal to the required capacity $R_{k}$ it is claiming. And so, each $\mathrm{B}-\mathrm{AP}$ which reaches its limit retires from the applicants side in order to give better chances for the remaining B-APs to fulfill their needs as well.

The DA algorithm user proposing for a limited allocation scheme is detailed in Algorithm 2.

In this case, before initiating the selection process, each B-AP ${ }_{k}$ fixes its own threshold to $R_{k}$. And so, the number 

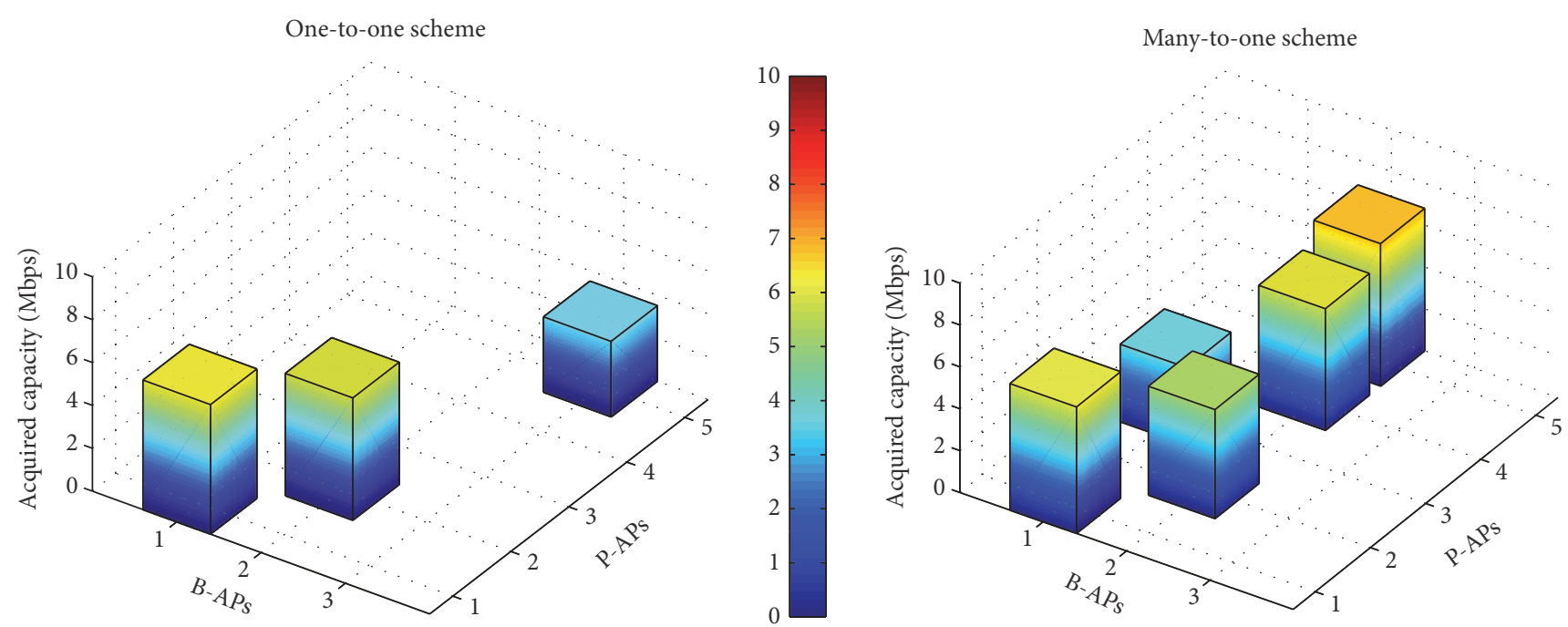

FIGURE 8: One-to-one versus many-to-one optimal mapping: “ $3 \times 5$ ”.

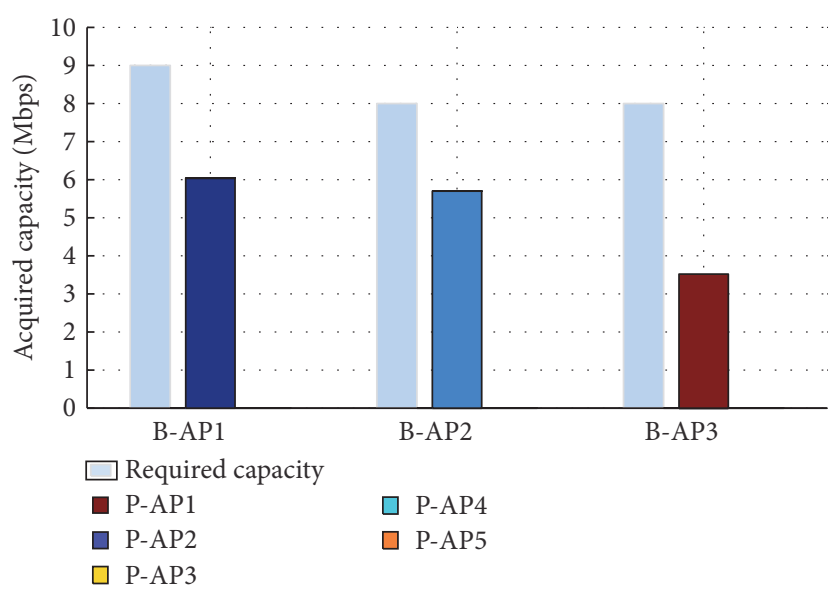

FIGURE 9: One-to-one matching: acquired capacity per B-AP, “ $3 \times 5$ ”.

of applications per B-AP (the number of iterations of the algorithm: i.e., Stage 3 ) is determined by the amount of $A B R$ $r_{k}$ it has acquired. Hence, if the limit $R_{k}$ of $\mathrm{B}_{-} \mathrm{AP}_{k}$ is achieved or exceeded, this latter stops applying for more ABR so that other B-APs, still in the game, will be left with more available resources.

We now compare the performance of greedy versus limited allocation modes in the context of many-to-one matching.

As shown before, resource abundance or scarcity impacts tremendously the system behavior with a one-to-one matching game. In the many-to-one allocation mode, however, abundant versus scarce distinction does not matter this much. With no restriction imposed on access resources, the same " $a \times b$ " configuration could be abundant for some B-APs and scarce for others. Here, the factor that matters the most is the order of preferences among the opposite sets.

As an illustrative example, we opt for a symmetric " $4 \times 4$ " configuration. Figure 12 shows one particular realization of a matching between P-APs and B-APs for both one-to-one

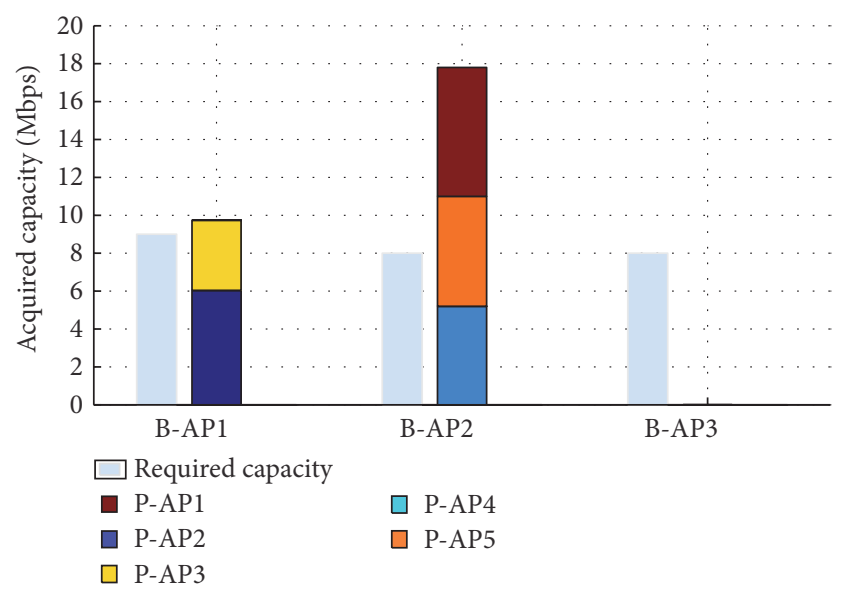

FIGURE 10: Many-to-one matching: acquired capacity per B-AP, “ $3 \times$ $5 "$.

and many-to-one allocation modes in a " $4 \times 4$ " configuration. Figures 13 and 14 show the individual performance in terms of achievable rate compared to the required capacity.

We observe a noticeable difference between the greedy versus limited resource allocation in terms of optimal matching and the related achievable backhaul rates $\left\{r_{k}\right\}_{k=1 \ldots 4}$. The greedy selection approach enables B-APs to greedily maximize their acquired capacity (e.g., B-AP3 with $250 \%$ more than its required capacity), while the limited access to resources ensures both a fair resource distribution among BAPs and better percentage of satisfaction (up to $75 \%$ of B-APs reach their thresholds).

Figure 15 shows slight difference in terms of bandwidth consumption. For the limited resource allocation, even with $R_{k}$ restriction, P-APs still retain the right to discard B-APs with lower SINR. This way, it could sustain signal strength and communication speed. And so, a limited resource distribution meets fairness requirement with close spectral efficiency level as for the greedy resource allocation. 


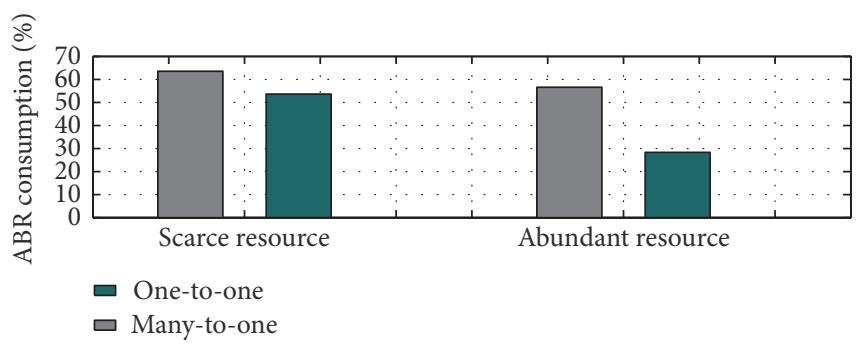

(a)

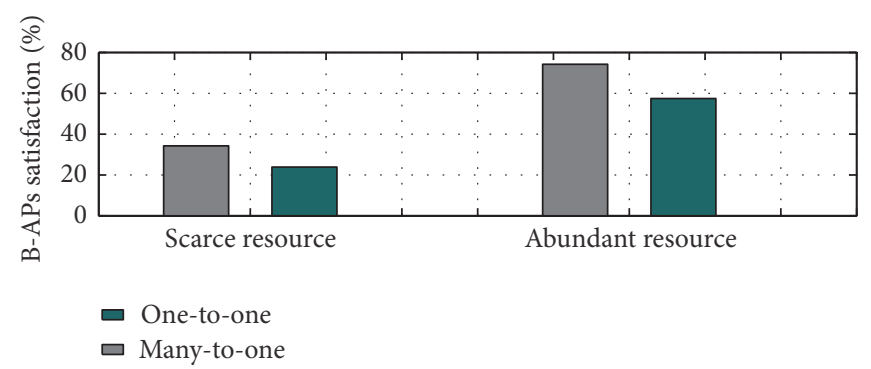

(b)

FIGURE 11: One-to-one versus many-to-one overall figures.
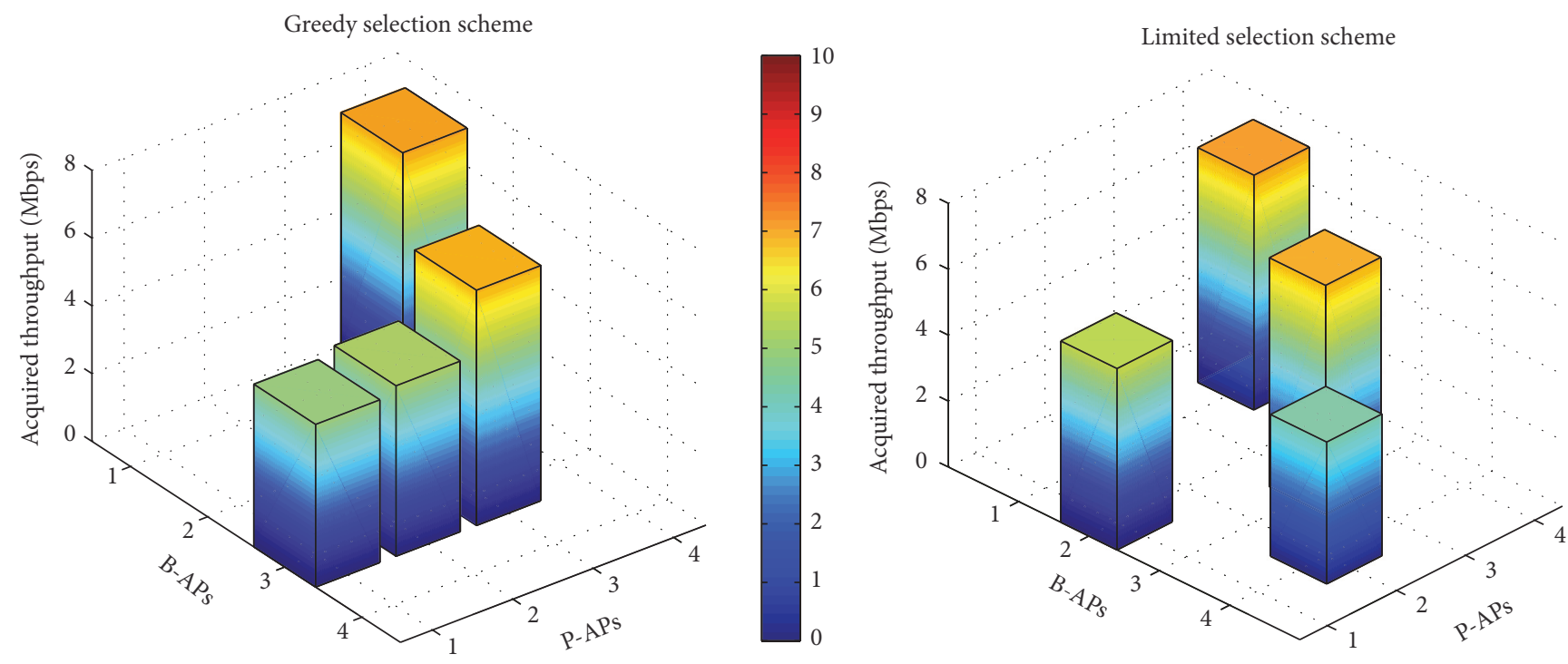

FIGURE 12: Greedy versus limited many-to-one mapping.

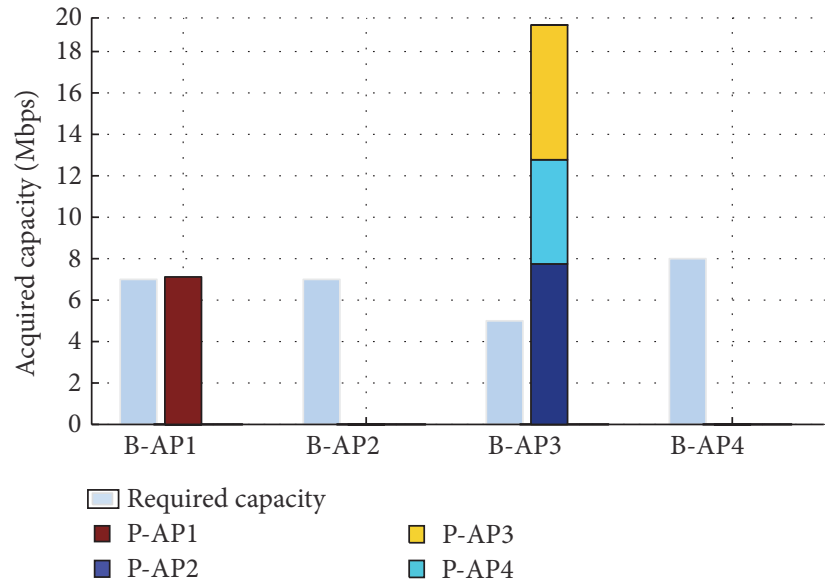

FIGURE 13: Greedy many-to-one: acquired capacity per B-AP “ $4 \times 4$ ”.

In most cases, the best strategy would be to satisfy the maximum number of APs within their limited threshold so as to satisfy most primary customers. The limited distribution is one way to reach more stable environment, balance load among B-APs, and spare larger shares of ABR.

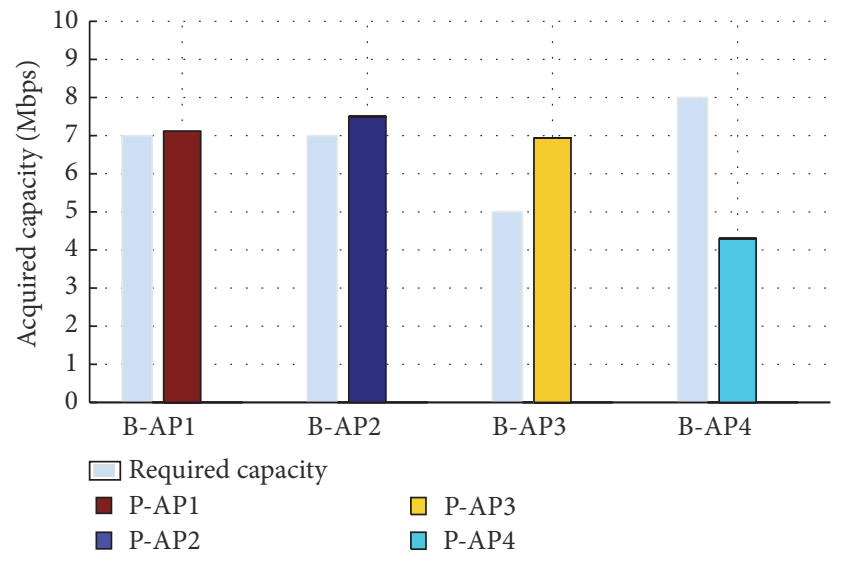

FIGURE 14: Limited many-to-one: acquired capacity per B-AP “ $4 \times 4$ ”.

To summarize, the limited many-to-one allocation outperforms the greedy one in terms of fairly distributed throughput gain and higher satisfaction percentage. Yet, it is noteworthy that some challenges could face our system even with such optimized approach. In some extreme cases, we 


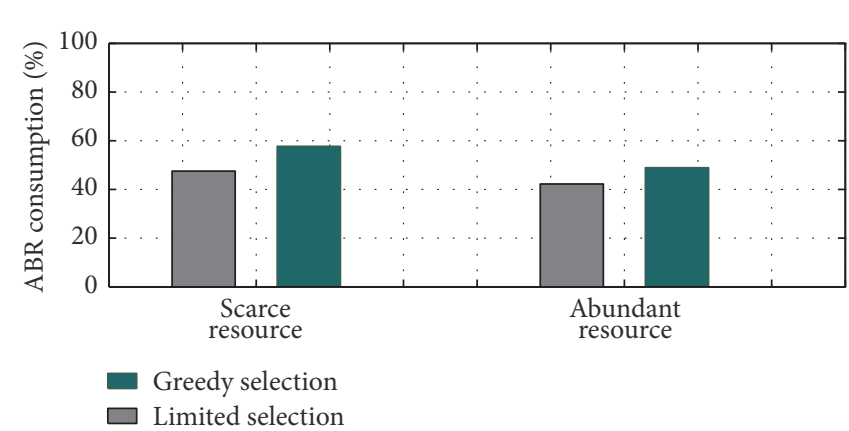

(a)

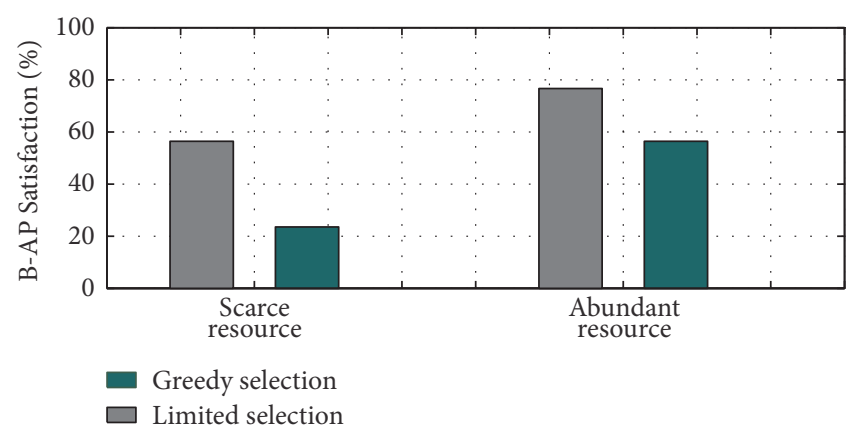

(b)

FIGURE 15: Greedy versus limited many-to-one overall figures.

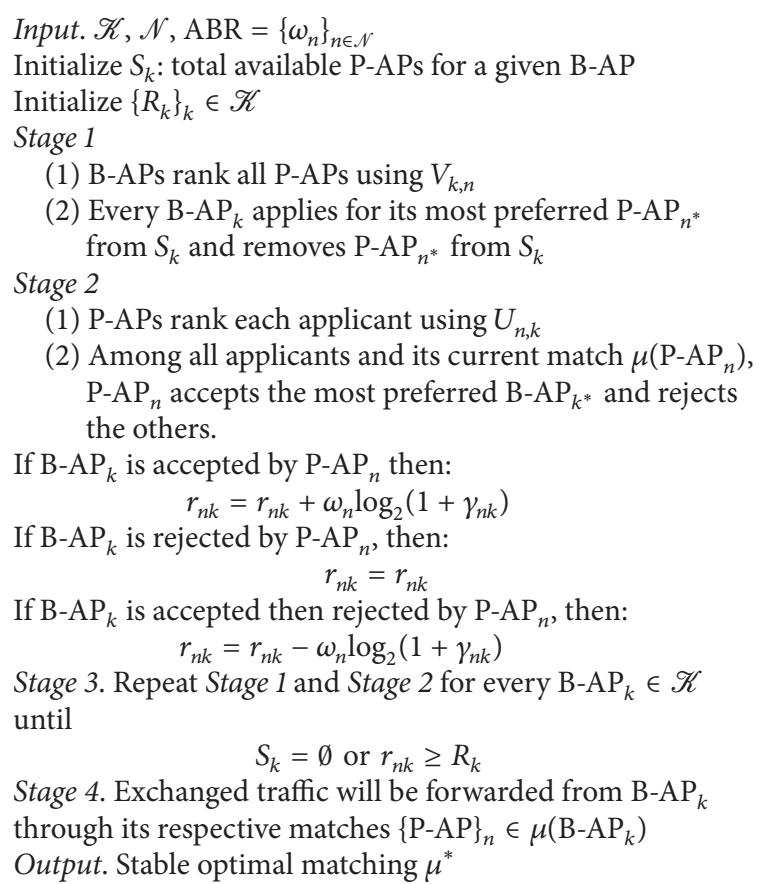

(1) B-APs rank all P-APs using $V_{k, n}$

(2) Every B-AP $\mathrm{P}_{k}$ applies for its most preferred P-AP $n^{*}$ from $S_{k}$ and removes $\mathrm{P}_{-} \mathrm{AP}_{n^{*}}$ from $S_{k}$

Stage 2

(1) P-APs rank each applicant using $U_{n, k}$

(2) Among all applicants and its current match $\mu\left(\mathrm{P}_{-} \mathrm{AP}_{n}\right)$, P-AP ${ }_{n}$ accepts the most preferred $\mathrm{B}-\mathrm{AP}_{k^{*}}$ and rejects the others.

If $\mathrm{B}-\mathrm{AP}_{k}$ is accepted by $\mathrm{P}-\mathrm{AP}_{n}$ then:

$$
r_{n k}=r_{n k}+\omega_{n} \log _{2}\left(1+\gamma_{n k}\right)
$$

If $\mathrm{B}^{-\mathrm{AP}_{k}}$ is rejected by $\mathrm{P}-\mathrm{AP}_{n}$, then:

$$
r_{n k}=r_{n k}
$$

If $\mathrm{B}-\mathrm{AP}_{k}$ is accepted then rejected by $\mathrm{P}-\mathrm{AP}_{n}$, then:

$$
r_{n k}=r_{n k}-\omega_{n} \log _{2}\left(1+\gamma_{n k}\right)
$$

Stage 3. Repeat Stage 1 and Stage 2 for every $\mathrm{B}_{-\mathrm{AP}_{k}} \in \mathscr{K}$ until

$$
S_{k}=\emptyset \text { or } r_{n k} \geq R_{k}
$$

Stage 4. Exchanged traffic will be forwarded from B-AP through its respective matches $\{\mathrm{P}-\mathrm{AP}\}_{n} \in \mu\left(\mathrm{B}-\mathrm{AP}_{k}\right)$

Output. Stable optimal matching $\mu^{*}$

Algorithm 2: DA algorithm user proposing: many-to-one limited resource allocation.

could end up in a greedy allocation case if one or more of B-APs ask for a required capacity $R_{k}$ that goes largely beyond required capacities' average; that is, $R_{k} \gg \operatorname{Avg}\left\{R_{j}\right\}_{j \in \mathscr{K} \backslash k}$. In this case, an alternate way to fix thresholds (based on traffic pattern, location history, operator preferences, etc.) could be envisaged.

\section{Conclusion}

In this paper, we proposed a distributed backhaul management approach for aggregating resources between providing and beneficiary APs in wireless local area networks. We formulated the problem as a many-to-one matching game and solved it using the DA algorithm obtaining a solution that is both optimal and stable.
Our simulation results showed that the many-to-one allocation scheme outperforms the one-to-one scheme in terms of throughput gain especially in the abundant resource case. Considering unlimited access to resources, the manyto-one matching ensures higher satisfaction percentage yet unbalanced resource assignment. The limited case provide, however, a more fair setting for the sharing of resources between beneficiaries as well as higher satisfaction levels.

As a future work, we intend to extend the work to the more complex case of many-to-many setting, where P-APs will be allowed to split their share of ABR to serve more than one B-AP at once.

\section{Conflicts of Interest}

The authors declare that there are no conflicts of interest regarding the publication of this paper.

\section{References}

[1] A. J. Nicholson, S. Wolchok, and B. D. Noble, "Juggler: virtual networks for fun and profit," IEEE Transactions on Mobile Computing, vol. 9, no. 1, pp. 31-43, 2010.

[2] E. Chai and K. G. Shin, "Sidekick: Ap aggregation over partially overlapping channels," in Proceedings of the IEEE International Conference on Network Protocols, pp. 301-310, Vancouver, Canada, October 2011.

[3] R. Chandra, P. Bahl, and P. Bahl, "Multinet: connecting to multiple IEEE 802.11 networks using a single wireless card," in Proceedings of the IEEE INFOCOM 2004 - Conference on Computer Communications - Twenty-Third Annual Joint Conference of the IEEE Computer and Communications Societies, pp. 882893, Hong Kong, China, March 2004.

[4] S. Kandula, K. C.-J. Lin, T. Badirkhanli, and D. Katab, "Fatvap: aggregating ap backhaul capacity to maximize throughput," in Proceedings of the USENIX Symposium on Networked Systems Design and Implementation, 2008.

[5] E. Goma and D. Giustiniano, "SmartAP: practical WLAN backhaul aggregation," in Proceedings of the 6th IFIP/IEEE Wireless Days Conference, WD 2013, Valencia, Spain, November 2013.

[6] J. Munkres, "Algorithms for the assignment and transportation problems," Journal of the Society for Industrial and Applied Mathematics, vol. 5, no. 1, pp. 32-38, 1957. 
[7] O. Semiari, W. Saad, Z. Dawy, and M. Bennis, "Matching theory for backhaul management in small cell networks with mmwave capabilities," in Proceedings of the IEEE International Conference on Communications, ICC 2015, pp. 3460-3465, London, UK, June 2015.

[8] K. Hassine, M. Frikha, and T. Chahed, "Proposal for access point backhaul resource aggregation and its modeling using one-to-one matching game," in Proceedings of the 13th International Conference on Advances in Mobile Computing and Multimedia, MoMM 2015, pp. 285-292, Brussels, Belgium, December 2015.

[9] Y. Gu, W. Saad, M. Bennis, M. Debbah, and Z. Han, "Matching theory for future wireless networks: Fundamentals and applications," IEEE Communications Magazine, vol. 53, no. 5, pp. 52-59, 2015.

[10] L. C. Liechty, Path loss measurements and model analysis of 2.4ghz wireless network [Ph.D. dissertation], Georgia Institute of Technology, 2007.

[11] C. E. Shannon, "Communication in the presence of noise," Proceedings of the IRE, 1998.

[12] F. Kelly, "Charging and rate control for elastic traffic," European Transactions on Telecommunications, vol. 8, no. 1, pp. 33-37, 1997.

[13] M. Vasef, "Effective capacity of a rayleigh fading channel in the presence of interference," International Journal of Wireless and Mobile Networks (IJWMN), vol. 4, no. 3, pp. 1-19, 2012.

[14] R. M. Karp, R. E. Miller, and J. W. Thatcher, "Reducibility among combinatorial problems," in Complexity of Computer Computations, University of California, Berkley, USA, 1972.

[15] A. E. Roth and M. Sotomayor, Hand Book for Game Theory, Elsevier Science Publisher, 1992.

[16] E. A. Jorswieck, "Stable matchings for resource allocation in wireless networks," in Proceedings of the 17th International Conference on Digital Signal Processing, DSP 2011, Corfu, Greece, July 2011.

[17] D. Gale and L. S. Shapley, "College admissions and the stability of marriage," American Mathematical Monthly, vol. 69, no. 1, pp. 9-15, 1962.

[18] K. Hassine, M. Frikha, and T. Chahed, "Greedy versus limited access point backhaul resource aggregation in wireless local area networks," in Proceedings of the International Conference on Advanced Information Networking and Applications, AINA, 2016. 


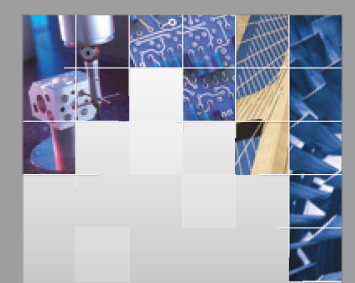

\section{Enfincering}
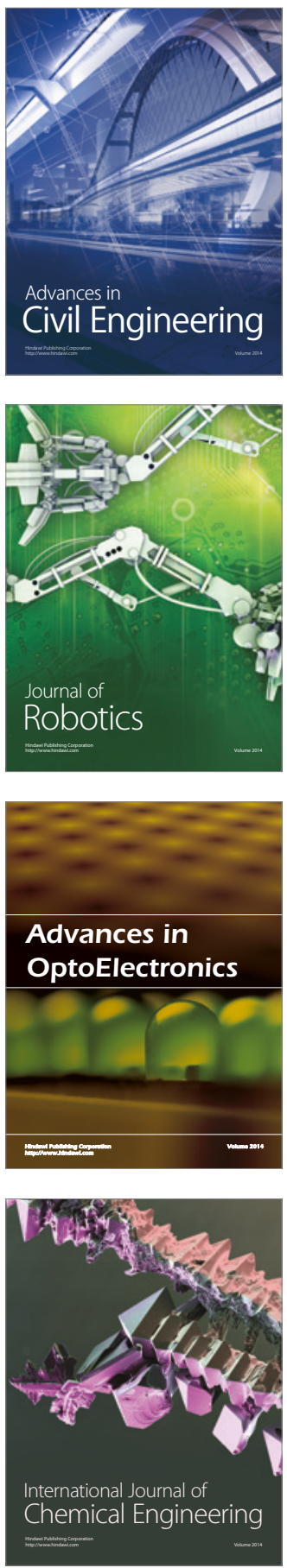

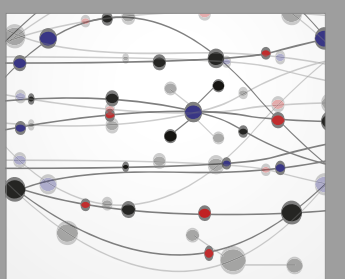

The Scientific World Journal

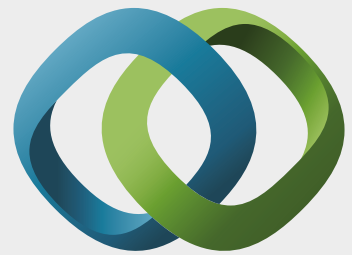

\section{Hindawi}

Submit your manuscripts at

https://www.hindawi.com
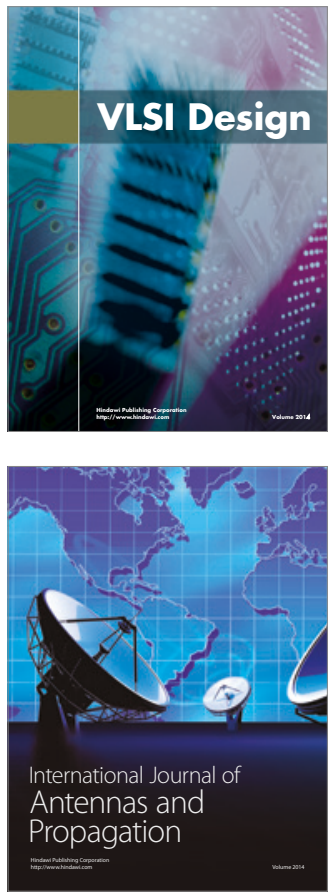

\section{Rotating}

Machinery
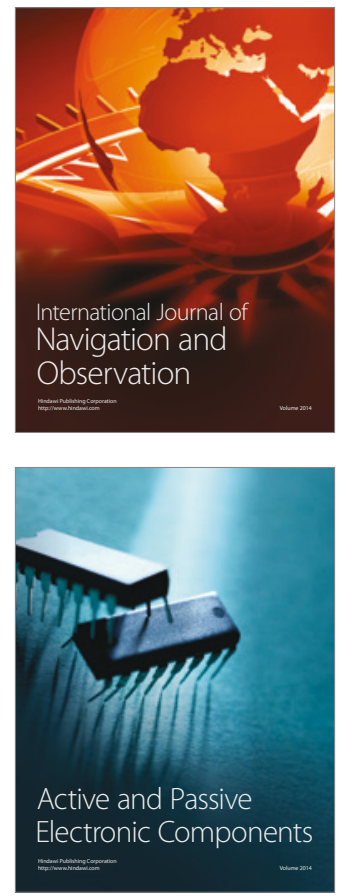
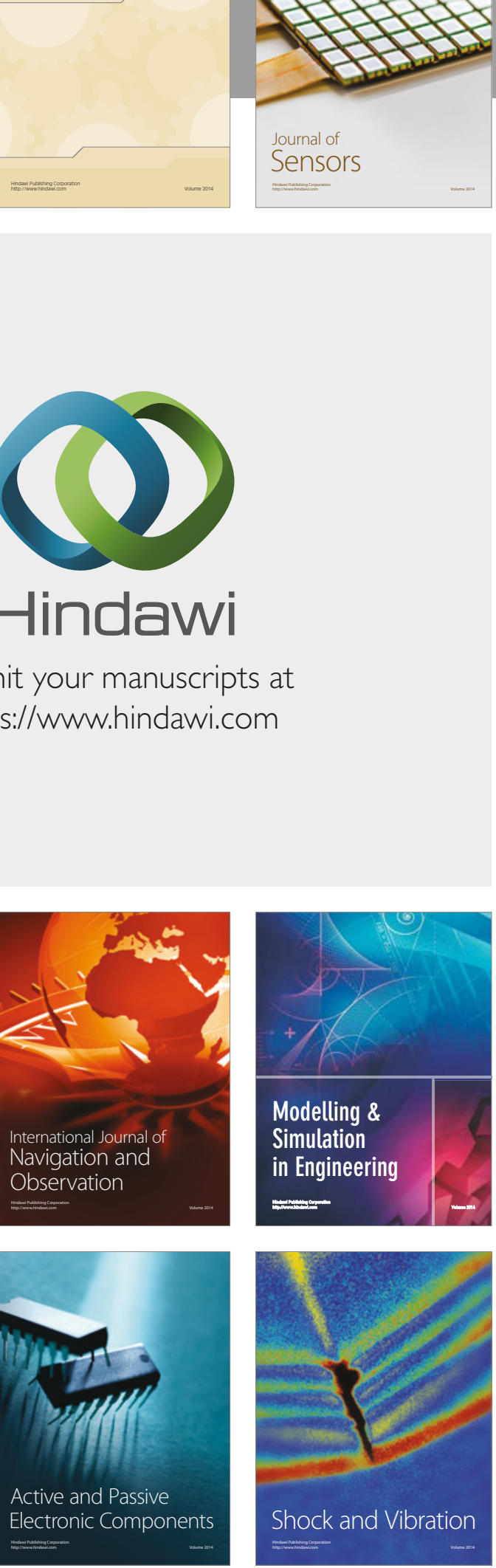
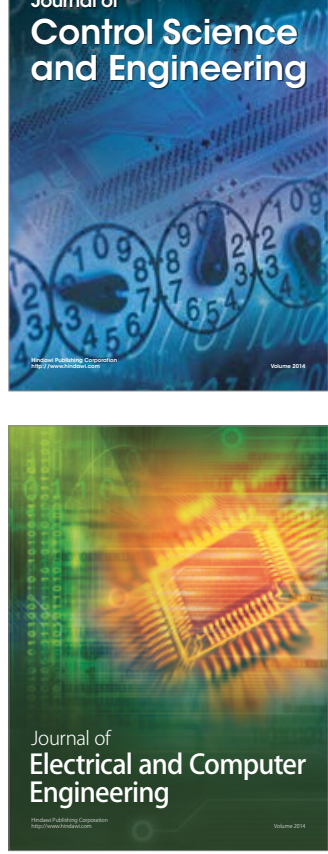

Distributed

Journal of

Control Science

and Engineering
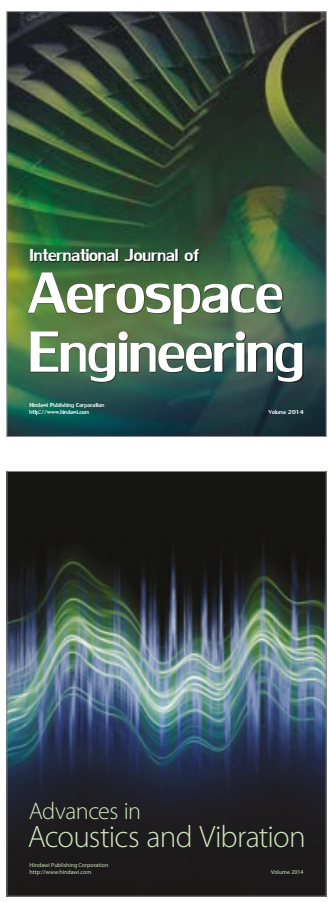

Sensor Networks 